\title{
Tunneling-tip-induced collapse of the charge gap in the excitonic insulator $\mathrm{Ta}_{2} \mathrm{NiSe}_{5}$
}

\author{
Qingyu He $\odot,{ }^{1, *}$ Xinglu Que, ${ }^{1}$ Lihui Zhou, ${ }^{1}$ Masahiko Isobe, ${ }^{1}$ Dennis Huang $\odot,{ }^{1}$ and Hidenori Takagi ${ }^{1,2,3, \dagger}$ \\ ${ }^{1}$ Max Planck Institute for Solid State Research, 70569 Stuttgart, Germany \\ ${ }^{2}$ Department of Physics, University of Tokyo, 113-0033 Tokyo, Japan \\ ${ }^{3}$ Institute for Functional Matter and Quantum Technologies, University of Stuttgart, 70569 Stuttgart, Germany
}

(Received 14 May 2021; revised 23 June 2021; accepted 8 September 2021; published 28 September 2021)

\begin{abstract}
Tuning many-body electronic phases by an external handle is of both fundamental and practical importance in condensed matter science. The tunability mirrors the underlying interactions, and gigantic electric, optical and magnetic responses to minute external stimuli can be anticipated in the critical region of phase change. The excitonic insulator is one of the more exotic phases of interacting electrons, produced by the Coulomb attraction between a small and equal number of electrons and holes, leading to the spontaneous formation of exciton pairs in narrow-gap semiconductors/semimetals. The layered chalcogenide $\mathrm{Ta}_{2} \mathrm{NiSe}_{5}$ has been recently discussed as a candidate for the excitonic insulator, though the nature of the excitation gap that opens below $T_{c}=328 \mathrm{~K}$ remains hotly debated. Here, we demonstrate a drastic collapse of the excitation gap in $\mathrm{Ta}_{2} \mathrm{NiSe}_{5}$ and the realization of a zero-gap state by moving the tip of a cryogenic scanning tunneling microscope towards the sample surface by a few angstroms. We argue that the collapse of the gap is driven predominantly by the electrostatic charge accumulation at the surface induced by the proximity of the tip and the resultant carrier doping. The fragility of the gap in the insulating state of $\mathrm{Ta}_{2} \mathrm{NiSe}_{5}$ strongly suggests the gap possesses many-body character. Our results establish a reversible phase-change function based on $\mathrm{Ta}_{2} \mathrm{NiSe}_{5}$.
\end{abstract}

DOI: 10.1103/PhysRevResearch.3.L032074

First proposed roughly 60 years ago, the excitonic insulator is an exotic phase of interacting electrons expected to arise in narrow-gap semiconductors and semimetals [1-3]. In narrowgap semiconductors, if the binding energy $E_{B}$ of electron-hole excitations due to the Coulomb attraction exceeds the singleelectron band gap $E_{G}$, then electron-hole pairs spontaneously condense below a transition temperature $T_{c}$. The state below $T_{c}$ is called an excitonic insulator and hosts elementary excitations characterized by a many-body gap $2 \Delta_{E}$, as conceptually illustrated in Fig. 1(a). When $E_{G}$ is approximately zero, $T_{c}$ and $2 \Delta_{E}$ are maximized, and when $E_{G}$ increases up to $E_{B}, T_{c}$ and $2 \Delta_{E}$ decrease to zero. Even in semimetals with $E_{G}<0$, the excitonic transition can still take place, but $T_{c}$ and $2 \Delta_{E}$ are suppressed with increasing band overlap $\left|E_{G}\right|$, since the attractive Coulomb interactions are rapidly screened out by the increasing number of holes and electrons. As a result, $T_{c}$ exhibits a dome-shaped dependence on $E_{G}$, with maximum at zero gap, $E_{G}=0$.

Recent intensive studies have established the ternary layered chalcogenide $\mathrm{Ta}_{2} \mathrm{NiSe}_{5}$ as one of the most promising candidates for an excitonic insulator in a bulk material [4-20].

\footnotetext{
*q.he@fkf.mpg.de

†h.takagi@fkf.mpg.de
}

Published by the American Physical Society under the terms of the Creative Commons Attribution 4.0 International license. Further distribution of this work must maintain attribution to the author(s) and the published article's title, journal citation, and DOI. Open access publication funded by the Max Planck Society.
$\mathrm{Ta}_{2} \mathrm{NiSe}_{5}$ is electronically quasi-one-dimensional, as each layer consists of an alternating array of one chain of $\mathrm{NiSe}_{4}$ tetrahedra and two chains of $\mathrm{TaSe}_{6}$ octahedra [Figs. 2(a) and 2(b)]. It has a nearly zero or negative direct gap at the $\Gamma$ point $[7,16,17]$, with Ta $5 d$ orbitals comprising the conduction bands and Ni $3 d$ ( $\mathrm{Se} 4 p$ ) orbitals comprising the valence bands. Below $T_{c}=328 \mathrm{~K}, \mathrm{Ta}_{2} \mathrm{NiSe}_{5}$ undergoes a phase transition to an insulator state with a finite excitation gap $[7,10]$. Two initial observations supported the excitonic insulator nature of this gap. First, optical conductivity data revealed the formation of a charge gap [7] with an optical gap edge of $0.16 \mathrm{eV}$ and an isosbestic point of gap opening $\sim 0.3 \mathrm{eV}$ below $T_{c}$. The energy scale of this gap agreed reasonably well with the exciton binding energy $E_{B} \sim 0.25 \mathrm{eV}$ estimated for the sister compound $\mathrm{Ta}_{2} \mathrm{NiS}_{5}$, which is a band insulator with a large single-electron energy gap of $0.6 \mathrm{eV}$ [8]. Second, upon application of pressure or sulfur substitution, $E_{G}$ could be controlled, and $T_{c}$ traced out a dome peaked at $E_{G} \sim 0$, corresponding to pure $\mathrm{Ta}_{2} \mathrm{NiSe}_{5}$ at ambient pressure [7].

Nevertheless, $\mathrm{Ta}_{2} \mathrm{NiSe}_{5}$ undergoes a structural transition from the orthorhombic to the monoclinic phase at $T_{c}=328 \mathrm{~K}$, without superlattice formation, concomitant with the electronic phase transition $[14,21,22]$. The hybridization of the Ta conduction bands and the Ni valence bands, which is forbidden by symmetry in the orthorhombic phase, is permitted in the monoclinic phase, giving rise to the possible opening of a one-electron gap. Recent symmetry analysis has shown that a finite excitonic order parameter must be accompanied by the monoclinic distortion [20]. This means that the hybridization gap, which is single electron in nature, is inherently and 


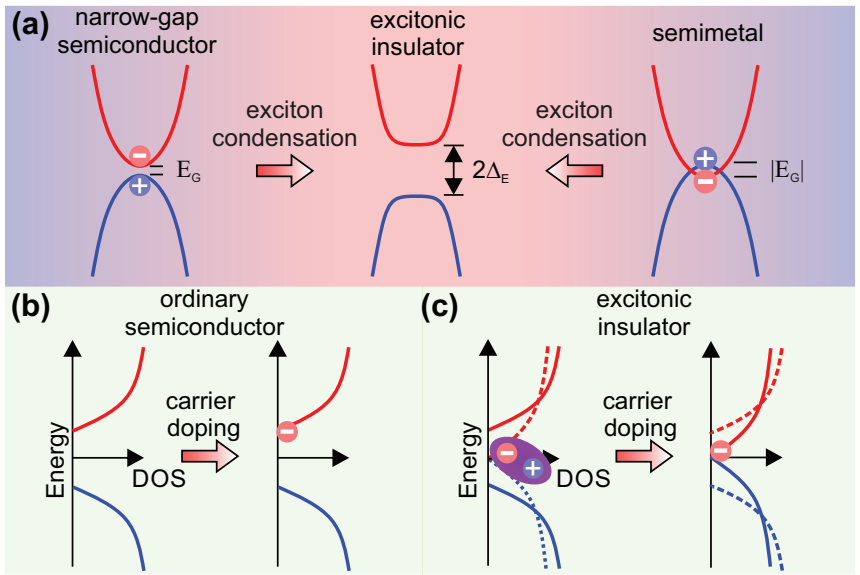

FIG. 1. (a) Conceptual image of the transition from a narrow-gap semiconductor (left) or semimetal (right) to an excitonic insulator (middle). A many-body gap $2 \Delta_{E}$ opens up in the excitonic phase. (b),(c) Doping of an ordinary semiconductor or an excitonic insulator leading to a rigid shift of the chemical potential or the collapse of the energy gap, respectively.

inevitably involved in the formation of the excitation gap [20,23-25]. Theoretical calculations for the monoclinic phase of $\mathrm{Ta}_{2} \mathrm{NiSe}_{5}$ predict a hybridization gap of up to $140 \mathrm{meV}$ $[23,24]$, clearly falling short of the experimental value of the charge gap. Therefore, the structural phase transition alone cannot fully explain the excitation gap, and its excitonic character deserves more attention.

Further hints may come from examining the fragility of the insulating phase. As a general example, Mott insulators derived from repulsive Coulomb interactions between electrons often collapse into a metallic phase with a small number of carrier doping and/or application of pressure, reflecting the many-body character of their charge gap. Such control of electronic phases provides the basis for correlated electronics [7,26-31]. The same concepts may be extended to manybody electronic systems with attractive interactions, namely, an excitonic insulator. Several optical pumping studies have been devoted to the tuning of the insulating phase $[12,13]$ in $\mathrm{Ta}_{2} \mathrm{NiSe}_{5}$ and an electronic phase evolution in the time domain has been observed. Angle-resolved photoemission spectroscopy (ARPES) measurements have also probed the effect of depositing $\mathrm{K}$ atoms on the surface of $\mathrm{Ta}_{2} \mathrm{NiSe}_{5}$, resulting in a Stark effect, carrier doping, or both $[16,17]$. As excitonic pairs consist of equal numbers of electrons and holes, we naturally expect that modest electron or hole doping would disturb the balance and thereby rapidly suppress the excitonic phase and the excitation gap. In contrast, if the gap were predominantly a single-electron hybridization gap, as in a conventional semiconductor, the doping effect would not modify the gap, but only shift the chemical potential, as shown in Fig. 1(b). Thus, more experiments along this line, perhaps by tuning the carrier density in a local, reversible, and clean manner, may illuminate whether exciton formation or hybridization due to the monoclinic distortion plays the primary role.

In this work, we utilized cryogenic scanning tunneling microscopy (STM) to trigger and to probe the phase change (a)

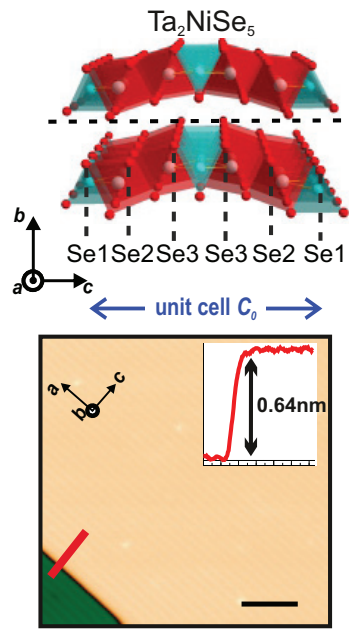

(d)

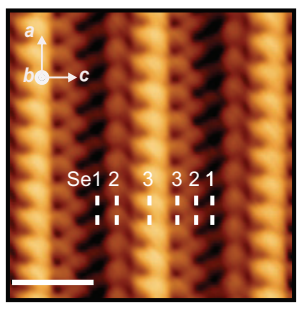

(b)

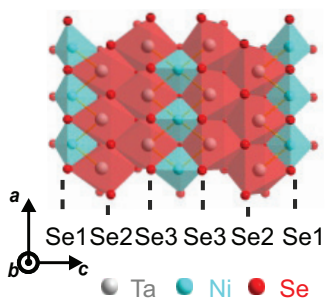

(e)

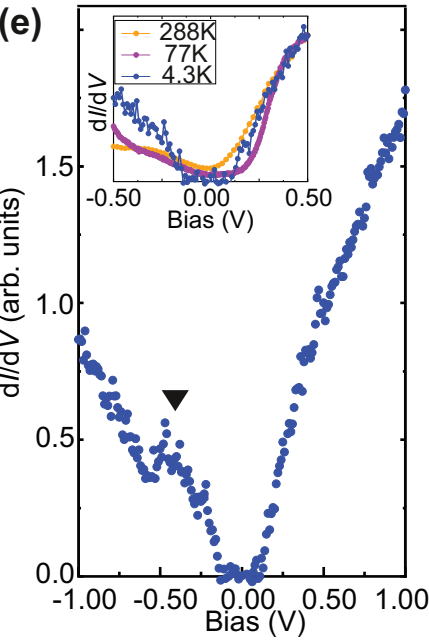

FIG. 2. (a),(b) Crystal structure of $\mathrm{Ta}_{2} \mathrm{NiSe}_{5}$ presented in two different views. The horizontal dashed line marks the cleavage plane. (c) Large-area topography of $\mathrm{Ta}_{2} \mathrm{NiSe}_{5}\left(53 \mathrm{~nm} \times 53 \mathrm{~nm}, I_{s}=\right.$ $100 \mathrm{pA}, V_{s}=400 \mathrm{mV}, T=77 \mathrm{~K}$ ). The insets show the correspondence to the crystallographic axes and the line-cut height profile through the step edge denoted by the red line. The black bar represents a length of $10 \mathrm{~nm}$. (d) Small-area topography of $\mathrm{Ta}_{2} \mathrm{NiSe}_{5}$ with atomic resolution $\left(3.5 \mathrm{~nm} \times 3.5 \mathrm{~nm}, I_{s}=1 \mathrm{nA}, V_{s}=400 \mathrm{mV}\right.$, $T=77 \mathrm{~K})$. The insets show the correspondence to the crystal structure and crystallographic axes. The white bar represents a length of $1 \mathrm{~nm}$. (e) Spectroscopic data taken at $4.3 \mathrm{~K}$, exhibiting a charge gap and a terracelike structure at negative biases, marked by the triangle. The inset displays the spectra taken at various temperatures, tracking the gap evolution.

in the excitonic insulator candidate $\mathrm{Ta}_{2} \mathrm{NiSe}_{5}$. We discovered an abrupt collapse of the excitation gap from $\sim 0.25 \mathrm{eV}$ to zero upon moving the STM tip towards the sample surface by merely a few angstroms. We argue that the phase change is driven by tip-induced electrostatic surface charges, which generate an imbalance of electron and hole densities and cause the system to revert to a zero-gap semiconductor, as schematically shown in Fig. 1(c). Our results strongly suggest that the gap in $\mathrm{Ta}_{2} \mathrm{NiSe}_{5}$ has many-body and excitonic character and demonstrate nanoscale, reversible phase-tuning in the compound.

Single crystals of $\mathrm{Ta}_{2} \mathrm{NiSe}_{5}$ were grown via the chemical vapor transport technique performed in a sealed quartz tube with $\mathrm{I}_{2}$ as the transport agent, as described in Ref. [21]. The transport and optical conductivity measurements clearly indicate the transition from a narrow-gap semiconductor/semimetal to an insulator at $T_{c}=328 \mathrm{~K}$, which we ascribed to an excitonic transition in our previous communications [7]. All experiments were conducted in a 
home-built ultrahigh vacuum (UHV) STM facility with a base temperature of $4.3 \mathrm{~K}$. The mounted crystal was cleaved at a low temperature $(\sim 200 \mathrm{~K})$ in the UHV chamber, then quickly transferred to the microscope head held at $4.3 \mathrm{~K}$. The topographic images were acquired in constant-current mode. The differential conductance $d I / d V$ was recorded using a standard lock-in technique, by adding a modulation voltage $V_{m}$ to a fixed bias $V_{s}$. All data were collected at $4.3 \mathrm{~K}$ unless otherwise specified.

The topographic STM image is fully consistent with the crystal structure determined for bulk single crystals [Figs. 2(a) and 2(b)] and indicates a high quality of surface for spectroscopic investigations. In the large-area image in Fig. 2(c), we observe atomically flat and clean terraces with a defect density less than $0.002 \mathrm{~nm}^{-2}(\sim 0.001$ per unit cell in the topmost layer) and an atomic step with a height of $\sim 0.6 \mathrm{~nm}$, which agrees well with the interlayer distance, a half of the out-of-plane lattice constant $b$ [Fig. 2(c)]. The cleaved surface should represent Se atoms located at the top of layer. In the expanded image in Fig. 2(d), we can clearly see Se atoms at the expected positions and a rippling of the layer. Five Se chains within one period of the rippling modulation can be identified: the two in-phase atomic chains on the ridge are assigned to $\mathrm{Se} 3$, the two neighboring chains are assigned to $\mathrm{Se}$, and their respective neighboring chains at the bottom of valley are assigned to $\mathrm{Se} 1$. Though not visible, the Ni chains below the surface Se atoms should run along the Se1 chain and between the two Se3 chains, while the Ta chains below should lie between the $\mathrm{Se} 1$ and $\mathrm{Se} 2$ chains and between the $\mathrm{Se} 2$ and $\mathrm{Se} 3$ chains.

Measurements of the local density of states (LDOS) via $d I / d V$ spectroscopy on the well-defined surface reveal the emergence of a charge gap upon lowering the temperature below $T_{c}$. At $288 \mathrm{~K}$, barely below $T_{c}=328 \mathrm{~K}$, only a V-shaped pseudogap feature with finite $d I / d V$ at the Fermi energy is observed [Fig. 2(e) inset]. The incomplete gap near $T_{c}$ can be ascribed to the combined effects of thermal and lifetime broadening, similar to those observed in superconductors. With further lowering of the temperature, however, a clear charge gap develops, and below $77 \mathrm{~K}$, a nearly temperatureindependent, full gap of $\sim 0.25 \mathrm{eV}$ is manifested [Fig. 2(e) inset]. We note that all the LDOS spectra in Fig. 2 were taken at relatively large tip-sample distances (estimated to be larger than $0.92 \mathrm{~nm}$ ), in order to minimize any effects from the tip. The spectra also did not depend appreciably on the lateral position of the tip in real space. The LDOS spectra obtained in this work agree well with those reported in preceding STM studies $[6,15]$. The apparent shape difference is due to Feenstra normalization [32,33], and the gap of $\sim 0.25 \mathrm{eV}$ is consistent with the optical gap reported in our previous work $[7,8]$. In the valence band region below the gap (negative bias voltage), an additional terracelike structure is superposed on the LDOS between roughly -0.2 to $-0.5 \mathrm{eV}$, which also was observed in an earlier study and ascribed to impurities [6]. Our spectra are taken in a clean area distant from impurity atoms and the terrace structure is observed independent of the location of the tip. As later discussed, we suggest that these features originate neither from band structure details nor impurity atoms, but from self-trapped states of tip-induced electrons.
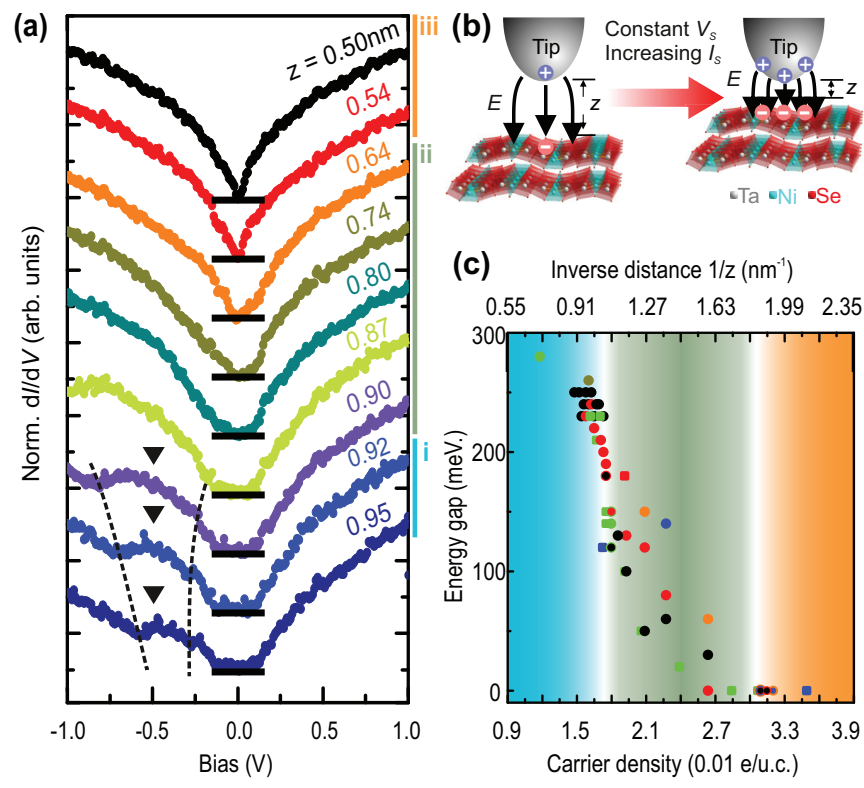

(c) Inverse distance $1 / \mathrm{z}\left(\mathrm{nm}^{-1}\right)$
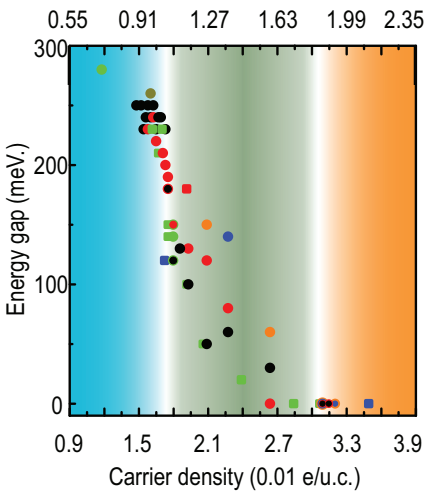

FIG. 3. (a) The conductance $d I / d V$ spectra taken with various tip-sample distances $z$ from 0.95 to $0.50 \mathrm{~nm}$. The $d I / d V$ curves are normalized by their maximum values and vertically shifted for clarity, with the horizontal black lines indicating the zero conductance for each curve. The terracelike structure discussed in the text is marked by the black triangles and the dashed lines. Three regimes(i) stable $0.25-\mathrm{eV}$ gap, (ii) gap shrinking, and (iii) stable zero gap — can be identified. (b) Schematic picture of the experimental setup. The tip-sample distance is controlled by varying the tunneling current $I_{s}$ while maintaining a fixed bias voltage $V_{s}$. The changing distance modifies the number of carriers induced by the difference in work function between the tip and the sample. (c) Experimentally determined charge gap as a function of the estimated surface carrier density and inverse distance. Different dataset are color and symbol coded: balls, $V_{s}=2 \mathrm{~V}$; squares, $V_{s}=1.5 \mathrm{~V}$.

Our main discovery is that the $0.25-\mathrm{eV}$ charge gap in $\mathrm{Ta}_{2} \mathrm{NiSe}_{5}$ collapses rapidly upon moving the STM tip a few angstroms toward the surface and is therefore rather fragile against an external perturbation. The tip-sample distance $z$ in the STM setup is precisely controlled by varying the tunneling current $I_{s}$ while fixing the tip-sample voltage $V_{s}$ [as schematically shown in Fig. 3(b)]. $I_{s}$ can be converted to a tip-sample distance $z$ following the procedure described in Refs. [32,34,35]. Figure 3(a) displays the $d I / d V$ spectra with various tip-sample distances $z$ and shows the collapse of the $0.25-\mathrm{eV}$ gap upon lowering $z$ from 0.95 to $0.50 \mathrm{~nm}$. When the tip is far from the surface $(\geqslant 0.92 \mathrm{~nm})$, the $d I / d V$ spectra consistently show a $z$-independent gap of $0.25 \mathrm{eV}$ and the perturbation by the tip does not influence the insulating ground state appreciably. In an intermediate $z$ range from 0.92 to $0.54 \mathrm{~nm}$, however, the gap is apparently and very rapidly suppressed from $0.25 \mathrm{eV}$ to almost zero. Eventually, with $z<0.54 \mathrm{~nm}$, the gap appears to collapse completely and a V-shaped LDOS, indicative of a zero-gap semiconducting state, emerges. The drastic change of LDOS upon reducing $z$ can be separated into those three regimes, as marked in Fig. 3(a). Another series of experiments also shows the same collapse of the gap with decreasing $z$ [32]. We note that the 
tip-sample distances here, $>0.5 \mathrm{~nm}$, is large enough to eliminate the role of mechanical pressure by the tip in the observed gap collapse.

The approach of the tip towards the sample is accompanied by a systematic growth of the terracelike feature observed around -0.2 to $-0.5 \mathrm{eV}$ in the $d I / d V$ spectra, starting at a large $z=0.95 \mathrm{~nm}$. This dramatic growth suggests that the terrace structure is not an intrinsic band structure feature of $\mathrm{Ta}_{2} \mathrm{NiSe}_{5}$, but something else very likely connected to the mechanism of gap collapse. While the gap does not change appreciably, the terrace spreads over a wider energy range as $z$ decreases to $0.92 \mathrm{~nm}$. Below $z=0.92 \mathrm{~nm}$, the high-energy tail of terrace extends further and even into the gap region, which appears to trigger the collapse of the gap.

We ascribe the observed collapse of the charge gap to a carrier-doping effect from tip-induced electrostatic charges at the sample surface [36]. When the tip is brought in close proximity to the sample surface, an electric field is generated between the two due to the difference in their work functions. We use the literature value of the tip work function $\phi_{T}=4.50 \mathrm{eV}$ [37] and extract the sample work function $\phi_{S}=$ $5.05 \mathrm{eV}$ from $I-z$ spectroscopy (Ref. [9] estimated $5.4 \mathrm{eV}$ ), leading to a difference of $0.55 \mathrm{eV}$. We argue that the resulting electric field traps electrons at the sample surface, which manifest as the terracelike structure, $0.2-0.5 \mathrm{eV}$ below the Fermi energy, in the $d I / d V$ spectrum at $z=0.95 \mathrm{~nm}$. The fact that the terrace appears only below the Fermi energy implies that electrons (not holes) are trapped at the sample surface, consistent with the sign of the work function difference. Furthermore, the energy scale of the terrace, which represents the binding energy of these trapped electrons, is comparable to the work function difference. The width of the terrace in $d I / d V$ then very likely corresponds to the energy dispersion of the trapped electrons. When $z$ decreases from $0.95 \mathrm{~nm}$, the electric field and the number of trapped electrons increase, thereby causing the bandwidth of the terrace structure to grow [Fig. 3(a)]. The terrace band eventually spreads into the bulk gap below $z=0.92 \mathrm{~nm}$ and local carrier doping is expected, whereby the gap begins to collapse. At $z=0.92 \mathrm{~nm}$, the difference of work function gives an electric field of $6.1 \times 10^{6} \mathrm{~V} / \mathrm{cm}$ right below the tip and the electrostatically induced surface carrier density is estimated to be $3.4 \times$ $10^{-2} e / \mathrm{nm}^{2}$, which corresponds $1.8 \times 10^{-2} e /($ unit cell) assuming that the trapped electrons are only at the topmost layer [32]. This number is comparable to the population of electrons and holes participating in the formation of the (excitonic) charge gap, estimated to be $3 \times 10^{-2} /$ (unit cell) [7]. However, it is likely that only a fraction of the trapped surface electrons, those that lie in the high-energy tail of terrace structure that has extended into the gap region, contribute to doping. This may also explain the pinning of the chemical potential at the bottom of the V-shaped valley, in between the conduction band and the valence band, in the zero-gap semiconductor region, $z<0.54 \mathrm{~nm}$.

Figure 3(c) summarizes the gap evolution in $\mathrm{Ta}_{2} \mathrm{NiSe}_{5}$ versus the tip distance and estimated surface charge density for several data sets. We note that the drastic gap collapse is emphatically distinct from tip-induced band bending, which would result in a modest increase, not decrease, of the apparent band gap size $[32,38]$. The instability of the $0.25-\mathrm{eV}$
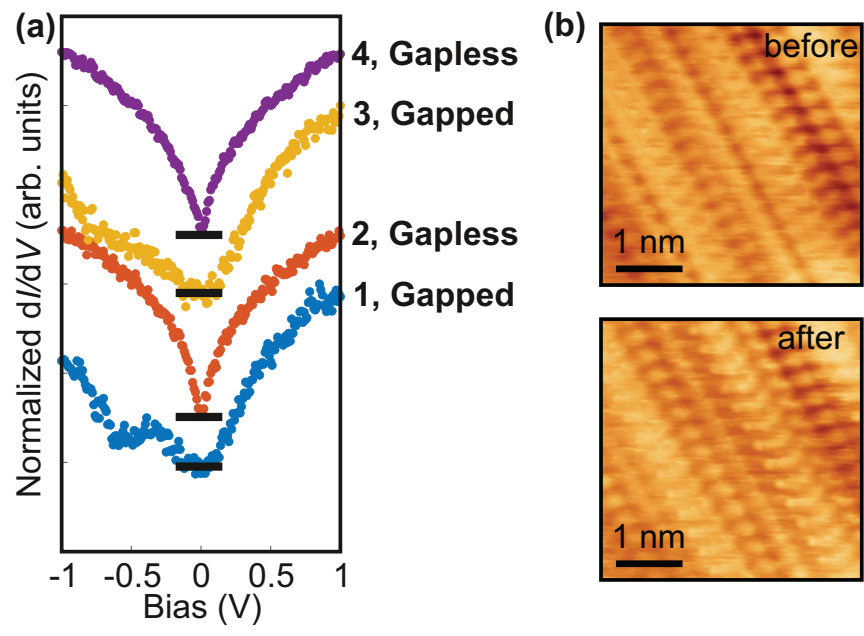

FIG. 4. (a) $d I / d V$ spectra showing the reversibility of the gap control, with 1 to 4 depicting the sequence of data acquisition. The spectra have been normalized by their maximum value and vertically offset for clarity, with the horizontal black lines marking the zero level for each curve. (b) Topography before and after the gap-control procedure.

excitation gap to an STM tip points to it having dominant excitonic insulating character. The addition of extra electrons to an excitonic insulator has a pair-breaking effect, since the extra electron would exert an oppositely repulsive/attractive force on a bound electron/hole pair. Similar to the case of magnetic impurities in a conventional superconductor or nonmagnetic impurities in an excitonic insulator [39], there would be a small critical concentration of extra electrons beyond which the excitonic insulating phase collapses.

The gap is fully closed for $z<0.54 \mathrm{~nm}$ and we observed a zero-gap semiconductor state. If there were no spontaneous structural phase transition accompanying the tip-induced gap collapse, i.e., $\mathrm{Ta}_{2} \mathrm{NiSe}_{5}$ stays in the monoclinic phase, our result would imply that the band hybridization allowed in the monoclinic state of $\mathrm{Ta}_{2} \mathrm{NiSe}_{5}$ is insufficient to open up any sizable single-particle gap and the gap in the low temperature phase is predominantly driven by the excitonic mechanism. Alternatively, there could be a local simultaneous structural transition back to the orthorhombic structure triggered by the tip-induced electronic transition, but we could not distinguish a loss of tiny monoclinic distortion in our STM images. If a structural transition did occur, our results would suggest that the "parent" orthorhombic state of $\mathrm{Ta}_{2} \mathrm{NiSe}_{5}$ is a zero-gap semiconductor. However, this appears to be inconsistent with ARPES measurements of $\mathrm{Ta}_{2} \mathrm{NiSe}_{5}$ in the high-temperature, orthorhombic state, which revealed a negative-gap semimetal [23].

Finally, scanning probe microscopy has proven to be a versatile tool in inducing nanoscale phase transitions in a wide range of strongly correlated materials, particularly Mott insulators. Other means of triggering local insulator-to-metal transitions include the use of voltage pulses in $1 T-\mathrm{TaS}_{2}$ [40,41] or cantilever-induced strain in $\mathrm{V}_{2} \mathrm{O}_{3}$ [42]. We note some distinguishing features of our tip-induced effect: Unlike the case of $1 T-\mathrm{TaS}_{2}$, the zero-gap semiconducting state in $\mathrm{Ta}_{2} \mathrm{NiSe}_{5}$ is not metastable; we continuously tune back and 
forth between the gapped and zero-gap states with the tip distance, as shown in Fig. 4(a). Unlike the case of $\mathrm{V}_{2} \mathrm{O}_{3}$, we avoid mechanical contact, as evidenced in the identical topographic images before and after the gap tuning [Fig. 4(b)], revealing no signs of tip or sample damage. The gap control in $\mathrm{Ta}_{2} \mathrm{NiSe}_{5}$ is nanoscale, noninvasive, and reversible. Overall, these systems highlight the fragility of many-body gaps and their susceptibility to tuning via local probes. By comparing these works, it appears that the phase transition in the excitonic insulator candidate $\mathrm{Ta}_{2} \mathrm{NiSe}_{5}$ is more easily triggered than in the Mott insulators $1 T-\mathrm{TaS}_{2}$ and $\mathrm{V}_{2} \mathrm{O}_{3}$, which require voltage pulses or cantilever-induced pressure.

In conclusion, in our spectroscopic STM measurements of the putative excitonic insulator $\mathrm{Ta}_{2} \mathrm{NiSe}_{5}$, we discovered a collapse of the $0.25-\mathrm{eV}$ excitation gap in the LDOS upon moving the STM tip towards the surface by only a few tenths of nanometers. We argue that the fragility of the $0.25-\mathrm{eV}$ gap to the addition of a small amount of carriers points to a dominant many-body, excitonic insulating character. Our results also demonstrate a local and reversible control of the charge gap in $\mathrm{Ta}_{2} \mathrm{NiSe}_{5}$, which may be detected electronically and optically. This effect may form the basis of nanoscale phase-change devices with memory and sensor functions, wherein the insulating gap, not the carrier density, can be tuned, analogous to the case for a Mott transistor with a fragile many-body gap.

The authors thank A. Rost, T. Takayama, A. Yaresko, U. Wedig, M. Daghofer, and S. Loth for discussion and comments, and K. Pflaum and M. Dueller for technical assistance. This work has been supported in part by the Alexander von Humboldt Foundation.
[1] N. F. Mott, The transition to the metallic state, Philos. Mag. 6, 287 (1961).

[2] W. Kohn, Excitonic Phases, Phys. Rev. Lett. 19, 439 (1967).

[3] D. Jérome, T. M. Rice, and W. Kohn, Excitonic insulator, Phys. Rev. 158, 462 (1967).

[4] Y. Wakisaka, T. Sudayama, K. Takubo, T. Mizokawa, M. Arita, H. Namatame, M. Taniguchi, N. Katayama, M. Nohara, and H. Takagi, Excitonic Insulator State in $\mathrm{Ta}_{2} \mathrm{NiSe}_{5}$ Probed by Photoemission Spectroscopy, Phys. Rev. Lett. 103, 026402 (2009).

[5] T. Kaneko, T. Toriyama, T. Konishi, and Y. Ohta, Orthorhombic-to-monoclinic phase transition of $\mathrm{Ta}_{2} \mathrm{NiSe}_{5}$ induced by the bose-einstein condensation of excitons, Phys. Rev. B 87, 035121 (2013).

[6] S. Y. Kim, Y. Kim, C.-J. Kang, E.-S. An, H. K. Kim, M. J. Eom, M. Lee, C. Park, T.-H. Kim, H. C. Choi, B. I. Min, and J. S. Kim, Layer-confined excitonic insulating phase in ultrathin $\mathrm{Ta}_{2} \mathrm{NiSe}_{5}$ crystals, ACS Nano 10, 8888 (2016).

[7] Y. F. Lu, H. Kono, T. I. Larkin, A. W. Rost, T. Takayama, A. V. Boris, B. Keimer, and H. Takagi, Zero-gap semiconductor to excitonic insulator transition in $\mathrm{Ta}_{2} \mathrm{NiSe}_{5}$, Nat. Commun. 8, 14408 (2017).

[8] T. I. Larkin, A. N. Yaresko, D. Pröpper, K. A. Kikoin, Y. F. Lu, T. Takayama, Y.-L. Mathis, A. W. Rost, H. Takagi, B. Keimer, and A. V. Boris, Giant exciton Fano resonance in quasi-one-dimensional $\mathrm{Ta}_{2} \mathrm{NiSe}_{5}$, Phys. Rev. B 95, 195144 (2017).

[9] S. Mor, M. Herzog, D. Golež, P. Werner, M. Eckstein, N. Katayama, M. Nohara, H. Takagi, T. Mizokawa, C. Monney, and J. Stähler, Ultrafast Electronic Band Gap Control in an Excitonic Insulator, Phys. Rev. Lett. 119, 086401 (2017).

[10] Y.-S. Seo, M. J. Eom, J. S. Kim, C.-J. Kang, B. I. Min, and J. Hwang, Temperature-dependent excitonic superfluid plasma frequency evolution in an excitonic insulator, $\mathrm{Ta}_{2} \mathrm{NiSe}_{5}$, Sci. Rep. 8, 11961 (2018).

[11] D. Werdehausen, T. Takayama, M. Höppner, G. Albrecht, A. W. Rost, Y. Lu, D. Manske, H. Takagi, and S. Kaiser, Coherent order parameter oscillations in the ground state of the excitonic insulator $\mathrm{Ta}_{2} \mathrm{NiSe}_{5}$, Sci. Adv. 4, eaap8652 (2018).
[12] S. Mor, M. Herzog, J. Noack, N. Katayama, M. Nohara, H. Takagi, A. Trunschke, T. Mizokawa, C. Monney, and J. Stähler, Inhibition of the photoinduced structural phase transition in the excitonic insulator $\mathrm{Ta}_{2} \mathrm{NiSe}_{5}$, Phys. Rev. B 97, 115154 (2018).

[13] K. Okazaki, Y. Ogawa, T. Suzuki, T. Yamamoto, T. Someya, S. Michimae, M. Watanabe, Y. Lu, M. Nohara, H. Takagi, N. Katayama, H. Sawa, M. Fujisawa, T. Kanai, N. Ishii, J. Itatani, T. Mizokawa, and S. Shin, Photo-induced semimetallic states realised in electron-hole coupled insulators, Nat. Commun. 9, 4322 (2018).

[14] A. Nakano, T. Hasegawa, S. Tamura, N. Katayama, S. Tsutsui, and H. Sawa, Antiferroelectric distortion with anomalous phonon softening in the excitonic insulator $\mathrm{Ta}_{2} \mathrm{NiSe}_{5}$, Phys. Rev. B 98, 045139 (2018).

[15] J. Lee, C.-J. Kang, M. J. Eom, J. S. Kim, B. I. Min, and H. W. Yeom, Strong interband interaction in the excitonic insulator phase of $\mathrm{Ta}_{2} \mathrm{NiSe}_{5}$, Phys. Rev. B 99, 075408 (2019).

[16] K. Fukutani, R. Stania, J. Jung, E. F. Schwier, K. Shimada, C. I. Kwon, J. S. Kim, and H. W. Yeom, Electrical Tuning of the Excitonic Insulator Ground State of $\mathrm{Ta}_{2} \mathrm{NiSe}_{5}$, Phys. Rev. Lett. 123, 206401 (2019).

[17] L. Chen, T. T. Han, C. Cai, Z. G. Wang, Y. D. Wang, Z. M. Xin, and Y. Zhang, Doping-controlled transition from excitonic insulator to semimetal in $\mathrm{Ta}_{2} \mathrm{NiSe}_{5}$, Phys. Rev. B 102, 161116(R) (2020).

[18] P. A. Volkov, M. Ye, H. Lohani, I. Feldman, A. Kanigel, and G. Blumberg, Critical charge fluctuations and emergent coherence in a strongly correlated excitonic insulator, npj Quantum Mater. 6, 52 (2020).

[19] M.-J. Kim, A. Schulz, T. Takayama, M. Isobe, H. Takagi, and S. Kaiser, Phononic soft mode behavior and a strong electronic background across the structural phase transition in the excitonic insulator $\mathrm{Ta}_{2} \mathrm{NiSe}_{5}$, Phys. Rev. Research 2, 042039(R) (2020).

[20] G. Mazza, M. Rösner, L. Windgätter, S. Latini, H. Hübener, A. J. Millis, A. Rubio, and A. Georges, Nature of Symmetry Breaking at the Excitonic Insulator Transition: $\mathrm{Ta}_{2} \mathrm{NiSe}_{5}$, Phys. Rev. Lett. 124, 197601 (2020). 
[21] F. Di Salvo, C. Chen, R. Fleming, J. Waszczak, R. Dunn, S. Sunshine, and J. A. Ibers, Physical and structural properties of the new layered compounds $\mathrm{Ta}_{2} \mathrm{NiS}_{5}$ and $\mathrm{Ta}_{2} \mathrm{NiSe}_{5}$, J. Less-Common Met. 116, 51 (1986).

[22] S. A. Sunshine and J. A. Ibers, Structure and physical properties of the new layered ternary chalcogenides tantalum nickel sulfide $\left(\mathrm{Ta}_{2} \mathrm{NiS}_{5}\right)$ and tantalum nickel selenide $\left(\mathrm{Ta}_{2} \mathrm{NiSe}_{5}\right)$, Inorg. Chem. 24, 3611 (1985).

[23] M. D. Watson, I. Marković, E. A. Morales, P. Le Fèvre, M. Merz, A. A. Haghighirad, and P. D. C. King, Band hybridization at the semimetal-semiconductor transition of $\mathrm{Ta}_{2} \mathrm{NiSe}_{5}$ enabled by mirror-symmetry breaking, Phys. Rev. Research 2, 013236 (2020).

[24] A. Subedi, Orthorhombic-to-monoclinic transition in $\mathrm{Ta}_{2} \mathrm{NiSe}_{5}$ due to a zone-center optical phonon instability, Phys. Rev. Mater. 4, 083601 (2020).

[25] E. Baldini, A. Zong, D. Choi, C. Lee, M. H. Michael, L. Windgaetter, I. I. Mazin, S. Latini, D. Azoury, B. Lv, A. Kogar, Y. Wang, Y. Lu, T. Takayama, H. Takagi, A. J. Millis, A. Rubio, E. Demler, and N. Gedik, The spontaneous symmetry breaking in $\mathrm{Ta}_{2} \mathrm{NiSe}_{5}$ is structural in nature, arXiv:2007.02909.

[26] H. Takagi and H. Y. Hwang, An emergent change of phase for electronics, Science 327, 1601 (2010).

[27] C. H. Ahn, J.-M. Triscone, and J. Mannhart, Electric field effect in correlated oxide systems, Nature (London) 424, 1015 (2003).

[28] C. H. Ahn, A. Bhattacharya, M. Di Ventra, J. N. Eckstein, C. D. Frisbie, M. E. Gershenson, A. M. Goldman, I. H. Inoue, J. Mannhart, A. J. Millis, A. F. Morpurgo, D. Natelson, and J.-M. Triscone, Electrostatic modification of novel materials, Rev. Mod. Phys. 78, 1185 (2006).

[29] J. T. Ye, Y. J. Zhang, R. Akashi, M. S. Bahramy, R. Arita, and Y. Iwasa, Superconducting dome in a gate-tuned band insulator, Science 338, 1193 (2012).

[30] J. M. Lu, O. Zheliuk, I. Leermakers, N. F. Q. Yuan, U. Zeitler, K. T. Law, and J. T. Ye, Evidence for two-dimensional ising superconductivity in gated $\mathrm{MoS}_{2}$, Science 350, 1353 (2015).

[31] Y. Deng, Y. Yu, Y. Song, J. Zhang, N. Z. Wang, Z. Sun, Y. Yi, Y. Z. Wu, S. Wu, J. Zhu, J. Wang, X. H. Chen, and Y. Zhang, Gate-tunable room-temperature ferromagnetism in twodimensional $\mathrm{Fe}_{3} \mathrm{GeTe}_{2}$, Nature (London) 563, 94 (2018).
[32] See Supplemental Material at http://link.aps.org/supplemental/ 10.1103/PhysRevResearch.3.L032074 for methods, additional data, and additional discussions.

[33] J. A. Stroscio, R. M. Feenstra, and A. P. Fein, Electronic Structure of the Si(111) $2 \times 1$ Surface by Scanning-Tunneling Microscopy, Phys. Rev. Lett. 57, 2579 (1986).

[34] J. M. Blanco, F. Flores, and R. Pérez, STM-theory: Image potential, chemistry and surface relaxation, Prog. Surf. Sci. 81 403 (2006).

[35] J. L. Collins, A. Tadich, W. Wu, L. C. Gomes, J. N. B Rodrigues, C. Liu, J. Hellerstedt, H. Ryu, S. Tang, S.-K. Mo, S. Adam, S. A. Yang, M. S. Fuhrer, and M. T. Edmonds, Electric-field-tuned topological phase transition in ultrathin $\mathrm{Na}_{3} \mathrm{Bi}$, Nature 564, 390 (2018).

[36] Y. Jiang, J. Mao, D. Moldovan, M. R. Masir, G. Li, K. Watanabe, T. Taniguchi, F. M. Peeters, and E. Y. Andrei, Tuning a circular $\mathrm{p}-\mathrm{n}$ junction in graphene from quantum confinement to optical guiding, Nat. Nanotechnol. 12, 1045 (2017).

[37] I. Battisti, V. Fedoseev, K. M. Bastiaans, A. de la Torre, R. S. Perry, F. Baumberger, and M. P. Allan, Poor electronic screening in lightly doped Mott insulators observed with scanning tunneling microscopy, Phys. Rev. B 95, 235141 (2017).

[38] F. Marczinowski, J. Wiebe, F. Meier, K. Hashimoto, and R. Wiesendanger, Effect of charge manipulation on scanning tunneling spectra of single Mn acceptors in InAs, Phys. Rev. B 77, 115318 (2008).

[39] J. Zittartz, Theory of the excitonic insulator in the presence of normal impurities, Phys. Rev. 164, 575 (1967).

[40] D. Cho, S. Cheon, K.-S. Kim, S.-H. Lee, Y.-H. Cho, S.-W Cheong, and H. W. Yeom, Nanoscale manipulation of the Mott insulating state coupled to charge order in $1 \mathrm{~T}-\mathrm{TaS}_{2}$, Nat. Commun. 7, 10453 (2016).

[41] L. Ma, C. Ye, Y. Yu, X. F. Lu, X. Niu, S. Kim, D. Feng, D. Tománek, Y.-W. Son, X. H. Chen, and Y. Zhang, A metallic mosaic phase and the origin of Mott-insulating state in $1 \mathrm{~T}-\mathrm{TaS}_{2}$, Nat. Commun. 7, 10956 (2016).

[42] N. Alyabyeva, J. Sakai, M. Bavencoffe, J. Wolfman, P. Limelette, H. Funakubo, and A. Ruyter, Metal-insulator transition in $\mathrm{V}_{2} \mathrm{O}_{3}$ thin film caused by tip-induced strain, Appl. Phys. Lett. 113, 241603 (2018). 